Temperature perturbation model of the opto-galvanic effect in $\mathrm{CO}_{2}$ laser discharges

This article has been downloaded from IOPscience. Please scroll down to see the full text article.

1984 J. Phys. D: Appl. Phys. 1759

(http://iopscience.iop.org/0022-3727/17/1/010)

View the table of contents for this issue, or go to the journal homepage for more

Download details:

IP Address: 130.159.17.137

The article was downloaded on $07 / 05 / 2010$ at $14: 56$

Please note that terms and conditions apply. 


\title{
Temperature perturbation model of the opto-galvanic effect in $\mathrm{CO}_{2}$ laser discharges
}

\author{
S Moffatt $†$ and A L S Smith \\ Department of Physics, University of Strathclyde, Glasgow G4 0NG, UK
}

Received 12 May 1983, in final form 2 August 1983

\begin{abstract}
A detailed discharge model of the opto-galvanic effect in molecular laser gas mixtures is developed based on the temperature perturbation or discharge cooling mechanism of Smith and Brooks (1979). Excellent agreement between the model and experimental results in $\mathrm{CO}_{2}$ laser gas mixtures is obtained. The model should be applicable to other molecular systems where the OGE is being used for laser stabilisation and as a spectroscopic tool.
\end{abstract}

\section{Introduction}

Although observations of the opto-galvanic effect (OGE) were made early in this century (Foote and Mohler 1925, Meissner and Graffunder 1927, Penning 1928), interest in the effect has only become widespread with the advent of tunable lasers and the use of the OGE for spectroscopic analysis (see recent reviews by Goldsmith and Lawler 1981 and Ferguson 1982). The OGE is most conveniently described as a change of the impedance of a gas discharge in response to the absorption or emission of radiation, although the effect is usually observed by measuring a change in the discharge current or voltage. Until recently almost all systems examined have been atomic and the effect is attributed to changes in the discharge multi-step ionisation rates due to stimulated emission or absorption altering the population of the intermediate metastable level.

The only detailed examination of the OGE in molecular systems has been in connection with the $\mathrm{CO}_{2}$ laser (see Smith and Brooks 1979, Moffatt and Smith 1981). Smith and Brooks (1979) concluded that the particularly large OGE in $\mathrm{CO}_{2}$ laser systems is due to gas number density changes caused by a change in the amount of gas heating from relaxing excited $\mathrm{CO}_{2}$ levels in $\mathrm{V}-\mathrm{T}$ and $\mathrm{V}-\mathrm{V}-\mathrm{T}$ processes as stimulated emission becomes an alternative vibrational energy loss channel. It is understandable that the principal OGE mechanism should be different in molecular discharges from that in atomic discharges. In atomic systems there are only relatively few (high-lying) energy levels participating in the discharge, and ionisation is the significant energy loss process. However in molecular gases there are many extra inelastic loss processes (e.g. rotational and vibrational excitation) and to maintain the discharges typically requires an order of magnitude greater voltage, resulting in very much greater gas heating. So any significant perturbation of the vibrational energy levels (such as that caused by a radiation field) is

$\div$ Present address: Applied Implant Technology, Horsham, UK. 
likely to significantly affect the total amount of $\mathrm{V}-\mathrm{T}$ energy transfer and hence the gas heating and number density.

We have previously established qualitatively (Smith and Moffatt 1979, Moffatt and Smith 1981) that in an electrically excited laser gas mixture, energy which would have been transferred from the excited asymmetric stretch mode of $\mathrm{CO}_{2}$ to translational energy $T_{u}$, in the absence of stimulated emission, by the process

$$
\mathrm{CO}_{2}(001)+M \rightarrow \mathrm{CO}_{2}(n, m, o)+M+T_{u}
$$

(where $M$ represents the gas collision partners $\mathrm{CO}_{2}, \mathrm{~N}_{2}, \mathrm{CO}, \mathrm{He}, \mathrm{Xe}$ and $\mathrm{O}_{2}$ etc) is alternatively lost by the photon de-excitation process

$$
\mathrm{CO}_{2}(001) \rightarrow \mathrm{CO}_{2}(100)+h \nu
$$

and hence the gas will be cooled by $T_{u}$ for each stimulated emission. Smith and Brooks (1979) have shown that, under normal laser operating conditions, the major effect of the gas cooling is to increase the gas number density (in the irradiated part of the gas volume) and hence decrease the electron mean free path and mobility, resulting in a discharge impedance increase. In this paper we present a simple perturbation model which quantitatively relates the changes in the laser internal radiation field irradiance $I$ (or output beam power $P_{\text {out }}$ ) to the observed fluctuation $\Delta P$ in the electrical discharge power $P$.

\section{Power perturbation theory for $\mathrm{CO}_{2}$ laser oscillators}

The energy gained by each electron in the discharge positive column per second due to the accelerating electric field $E$ is $e E v_{\mathrm{d}}$ where $e$ is the electronic charge and $v_{\mathrm{d}}$ is the electron drift velocity. For an electron density of $n_{\mathrm{e}}\left(\right.$ electrons $\left.\mathrm{cm}^{-3}\right)$ the energy gained per second per unit volume of discharge is $E e v_{\mathrm{d}} n_{\mathrm{e}}$ or $E j$ where $j$ is the positive column current density.

Now consider the energy lost. This will be proportional to the collision rate $n_{\text {coll }}$ and the energy lost per collision $E_{\text {coll }}$. The energy lost per second is therefore given by

$$
P_{\text {coll }}=n_{\mathrm{e}} n_{\text {coll }} E_{\text {coll }} .
$$

Collision with molecules is the dominant energy loss process for electrons and assuming that the energy loss per collision is constant (a reasonable approximation, see Smith and Brooks 1979) then any change of electron energy loss is primarily due to a change in the number of collisions which occur. The number of collisions is directly proportional to the number density of all the possible collision partners $(n)$. Hence the fractional energy loss change is

$$
\Delta P_{\text {coll }} / P_{\text {coll }}=\Delta n / n
$$

where $\Delta n / n$ is the fractional number density change. For a $C w$ molecular discharge the electron energy gained from the accelerating electric field equals the energy lost by collisions. The fractional change in the discharge input power is given by

$$
\Delta P / P=\Delta P_{\text {coll } /} / P_{\text {coll }}=\Delta n / n \text {. }
$$

Assuming ideal gas behaviour, the gas pressure $(p)$ is related to number density $n$ by

$$
p=n k T
$$


and the total number density change $\mathrm{d} n$ by differentiating is

$$
\mathrm{d} n=\left.\frac{\partial n}{\partial p}\right|_{T} ^{\mathrm{d} p}+\left.\frac{\partial n}{\partial T}\right|_{p} ^{\mathrm{d} T} .
$$

If the non-irradiated volume is large compared to the laser irradiated volume then the pressure remains approximately constant, $\mathrm{d} p \approx 0$ and the first term of (7) is negligible compared to the second term, and

$$
\Delta n / n=-\Delta T / T
$$

where $\Delta T$ is the temperature change brought about by a number density change $\Delta n$.

Empirically it is found (Shields and Smith 1977 and unpublished) that the translational temperature for a laser discharge of this kind is given by

$$
T=t_{\mathrm{w}}+t_{\mathrm{dis}}=t_{\mathrm{w}}+m P / L
$$

where $t_{\mathrm{w}}$ is the wall temperature, $t_{\mathrm{dis}}$ the temperature rise due to dissipation of the discharge power, $P / L$ is the input power per unit length and $m$ is a coefficient dependent upon the gas pressure and composition. Measurement of $m$ is easily made with a simple small thermocouple technique and typical results of the variation of gas temperature with discharge current, $P / L$ and composition are given in Smith (1977).

In a laser oscillator as the stimulated emission removes $Q(\mathrm{~W})$ from the $\mathrm{CO}_{2}$ asymmetric stretch mode, translational energy is removed from the gas (via the $\mathrm{V}-\mathrm{V}-\mathrm{T}$ processes) and a fractional change in gas temperature occurs, given by

$$
\Delta T / T=-m Q / L\left(t_{\mathrm{w}}+m P / L\right)=-m Q /\left(L t_{\mathrm{w}}+m P\right)
$$

and the total energy removed by photons from the whole discharge is

$$
Q=V N h \nu
$$

where $N$ is the number density of photons generated by stimulated emission $\left(\mathrm{s}^{-1} \mathrm{~cm}^{-3}\right)$, and $V$ the irradiated discharge volume $(V=L A)$ is assumed to be uniformly radiating.

The value of $N$ is established by consideration of the field energy creation and loss when the $\mathrm{CO}_{2}$ laser irradiance $I\left(\mathrm{~W} \mathrm{~cm}^{-2}\right)$ is switched on optically, and thus

$$
N h \nu L A=I(\gamma / 2) A
$$

where the right-hand side represents the whole energy loss from the field and the loss factor $\gamma$ takes into account the output beam, reflection and absorption losses associated with the optics and loss due to the tube aperture.

Hence to summarise from equations (10), (11) and (12) the temperature fluctuation due to the beam irradiance $I$ is

$$
\Delta T / T=-A \gamma I / 2\left(L t_{\mathrm{w}} / m+P\right)
$$

and the accompanying electrical power input fluctuation ratio, $\Delta P / P$ due to the irradiance is in fractional terms

$$
\Delta P / P=\Delta n / n=-\Delta T / T=A \gamma I / 2\left(L t_{\mathrm{w}} / m+P\right)
$$

from equations (5), (8) and (13).

It is convenient to designate an OGE coefficient

$$
X=P / 2\left(L t_{\mathrm{w}} / m+P\right)=1 / 2\left(t_{\mathrm{w}} / t_{\mathrm{dis}}+1\right)
$$


and hence obtain the discharge input power fluctuation

$$
\Delta P=A X \gamma I \text {. }
$$

Alternatively the power fluctuation can be expressed in terms of the laser output beam power $P_{\text {out }}$ : for

$$
P_{\text {out }}=A I(1-R) /(1+R)
$$

where $R$ is the output coupler reflectivity. Then

$\Delta P=(1+R) \gamma P_{\text {out }} / 2(1-R)\left[L t_{\mathrm{w}} /(P m)+1\right]=[(1+R) /(1-R)] X \gamma P_{\text {out }}$.

\section{Comparison with experimental power perturbations}

\subsection{The laser system}

Comparison of actual observed laser discharge power perturbations with predictions based on the conclusions of $\S 2$ have been made using a number of lasers, but the majority of the results reported here have been obtained with the small sealed laser previously described (Smith and Moffatt 1979). A $8 \mathrm{~mm}$ bore water cooled quartz discharge tube ( $33 \mathrm{~cm}$ discharge length) is closed at one end by a $4 \mathrm{~m}$ radius of curvature gold coated copper reflector and at the other by a $\mathrm{ZnSe}$ or $\mathrm{KCl}$ Brewster window. The optical cavity is completed with a coupling mirror (usually $90 \%$ reflecting) mounted on a PZT stack rigidly attached to an Invar rod frame (mirror separation $\sim 44 \mathrm{~cm}$, discharge length in radiation field $25 \mathrm{~cm}$ ). The laser is operated either with passive stabilisation only, or with additional active OGE stabilisation as previously described. With a sealed filling of between 10 and 35 Torr of a $\mathrm{CO}_{2}-\mathrm{N}_{2}-\mathrm{He}-\mathrm{Xe}$ gas mixture (and a current of up to $40 \mathrm{~mA}$ ) a maximum power of about 8 watts is produced (at 15 to $20 \mathrm{~mA}$ ).

It has been previously shown (Smith and Brooks 1979) that if the power supply is operated in a constant voltage mode, analysis of the discharge perturbations will be considerably complicated for two reasons. First the constant voltage is at the power supply terminals, and current amplitude changes produced by the power supply to compensate for discharge impedance changes will produce a variation of potential difference across the series ballast resistor and hence the potential difference across the laser tube itself will not be constant. Second, there will be a perturbation amplification effect in the discharge gas itself: as the current changes with a radiation field induced impedance change, so the new current will produce a change of gas heating and a number density and hence a further impedance change will result. Thus the results presented here have been obtained with a power supply operating in the constant current mode and the results recorded after the system has settled to a new equilibrium after the application of the perturbation $(<1 / 10 \mathrm{~s}$ for a radiation field change, but a few seconds for a current change).

The laser power output has been measured with a CRL201 power meter and discharge voltage and voltage change with a 4 digit DVM and high voltage probe with input impedance $>100 \mathrm{M} \Omega$.

\subsection{Comparison of discharge and field perturbations}

It is convenient to compare the fluctuation in the discharge electrical input power $\Delta P$ with the change of laser output power $P_{\text {out }}$, rather than with the intra-cavity beam 
irradiance. The discharge input fluctuation $\Delta P$ is (at constant current) immediately calculable from $\Delta P=\mathrm{i} \Delta V$ where $\Delta V$ is the tube voltage change. Figure 1 shows the variation of $\Delta P$ with laser power level (obtained by changing the alignment of the laser output mirror and hence varying the internal cavity losses). Results are presented for a typical laser gas mixture and pressure (1 Torr Xe, 3 Torr $\mathrm{CO}_{2}, 4$ Torr $\mathrm{N}_{2}, 16$ Torr $\mathrm{He}$ ) and discharge currents from 4 to $40 \mathrm{~mA}$. The results suggest an approximate gradient of $\Delta P / P_{\text {out }}$ of 0.5 .

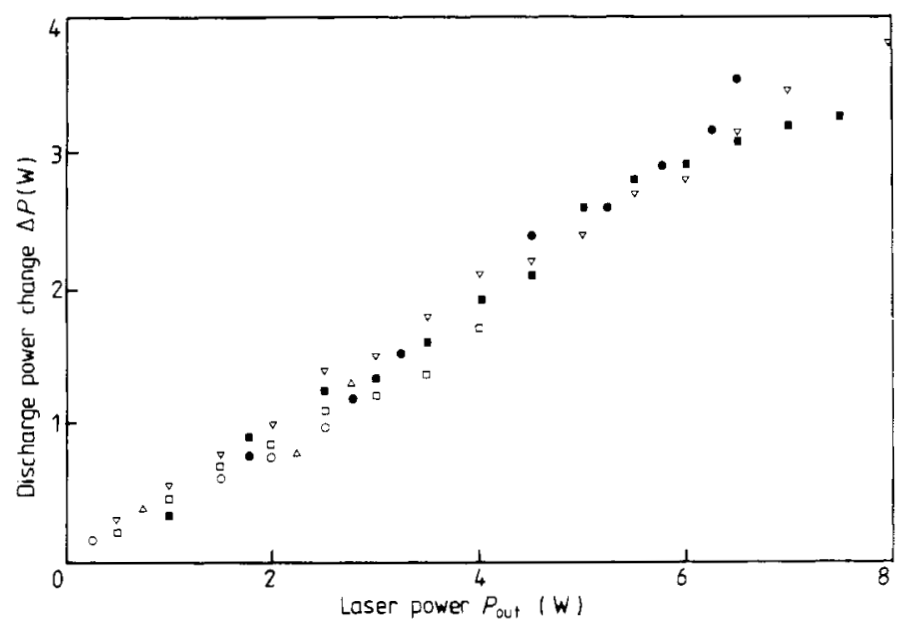

Figure 1. Variation of discharge power change with laser output power for discharge currents from 4 to $40 \mathrm{~mA}$ in a laser gas mixture of 1 Torr Xe, 3 Torr $\mathrm{CO}_{2}, 4$ Torr $\mathrm{N}_{2}, 16$ Torr $\mathrm{He}: \bigcirc$, $4 \mathrm{~mA} ; \square, 8 \mathrm{~mA} ; \nabla, 12 \mathrm{~mA} ; \mathbf{\square}, 16 \mathrm{~mA} ; \bigcirc, 30 \mathrm{~mA} ; \triangle, 40 \mathrm{~mA}$.

A preliminary numerical comparison can be made by reference to equation (18), the value of $\Delta P / P_{\text {out }}$ should depend not only on the values of the constants (output reflectivity $R=0.9$ and wall temperature $t_{\mathrm{w}} \sim 280 \mathrm{~K}$ ) but also on the extra gas temperature created by the discharge $\left(t_{\mathrm{dis}}=m P / L\right)$ and the total cavity losses $\gamma . \gamma$ is $\sim 0.15$ when the coupling mirror is fully aligned but can be increased to $\sim 0.35$ before oscillation ceases (with optimum excitation, see below), so with appropriate values of $t_{\text {dis }}$ (see $\$ 3.3$ below), $\Delta P / P_{\text {out }}$ is calculated to vary from $\sim 0.2$ at low currents to $\sim 0.8$ at high currents for an aligned cavity, and up to $\sim 1.1$ with a misaligned cavity. The mean experimental gradient of 0.5 lies well within the predicted range.

A closer comparison is obtained by examining the results for one particular discharge current near the optimum for good laser oscillation, say $10 \mathrm{~mA}$, which is obtained with a $P / L$ of $1.5 \mathrm{~W} \mathrm{~cm}^{-1}$ and for this gas mixture we have measured, using the thermocouple technique of Smith (1977), $t_{\text {dis }}$ to be $100 \pm 10 \mathrm{~K}$. When the laser is fully aligned the total losses are the output coupling $(1-R=0.1)$ and the sum of the Brewster window, fixed reflector, aperture and diffraction losses $(\sim 0.05-$ see $\$ 3.4$ below $)$, totalling $\sim 0.15$. The maximum value of $\gamma$ achieved before oscillation ceases depends on the round trip length $(2 \times 25 \mathrm{~cm})$ and the small signal gain. The small signal gain has been measured in a separate experiment by Smith and Mellis (1984), and we calculate the maximum $\gamma$ to be 0.35 (as the laser power decreases to zero).

Figure 2 shows the variation of the experimentally determined points $\Delta P$ with $P_{\text {out }}$ 
for a $10 \mathrm{~mA}$ discharge in 1 Torr $\mathrm{Xe}, 3$ Torr $\mathrm{CO}_{2}, 4$ Torr $\mathrm{N}_{2}$ and 17 Torr $\mathrm{He}$. The curves illustrate the theoretical predictions of equation (18) for three slightly different ranges of value of $\gamma$ (in all cases with the simplifying assumption that $\gamma$ varies linearly with $P_{\text {out }}$ ). Clearly reasonable agreement of the theoretical curves to the experimental points has been found, being best if the minimum cavity losses are taken to be 0.175 rather than 0.15 and the maximum cavity losses 0.30 rather than 0.35 .

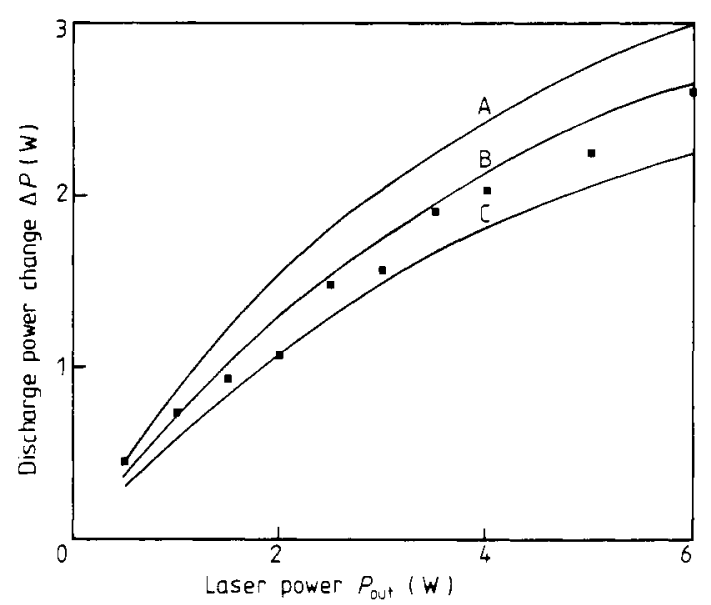

Figure 2. Variation of discharge power change with laser output power for a $10 \mathrm{~mA}$ discharge in 1 Torr Xe. 3 Torr $\mathrm{CO}_{2}, 4$ Torr $\mathrm{N}_{2}, 17$ Torr He. Solid curves show theoretical predictions for three ranges of total cavity losses $\gamma: \mathrm{A}, 0.35-0.20 ; \mathrm{B}, 0.30-0.175 ; \mathrm{C}, 0.25-0.15$.

It is convenient here to recall that in a previous publication we have noted an approximately linear relation between the discharge impedance fluctuation $\Delta Z / Z$ and the laser operating efficiency for constant current operation (see figure 4 of Smith and Moffatt 1979). Now at constant current $\Delta Z / Z$ is just the fractional power change $\Delta P / P$, which equation (18) gives as

$$
\Delta Z / Z=\Delta P / P=(1+R)\left[\gamma / 2(1-R)\left(t_{\mathrm{w}} / t_{\mathrm{dis}}+1\right)\right]\left(P_{\text {out }} / P\right)
$$

where of course $P_{\text {out }} / P$ is just the laser efficiency. With a suitable choice of constants (as in the paragraph above) the gradient of $(\Delta Z / Z) /($ efficiency) is calculated to be $\sim 0.5$ at high efficiencies (well aligned cavity) increasing to $\sim 1.0$ at low efficiency with a very misaligned cavity. On further inspection it can be seen that the results of figure 4 of Smith and Moffatt (1979) rather better fit a curve with such a slowly varying gradient, rather than a straight line.

\subsection{Temperature effects on the oGE coefficient}

In $\S 3.2$ we have seen that the size of the OGE is dependent on the magnitude of the radiation field perturbation, and in particular

$$
\Delta P / P_{\text {out }}=\{[(1+R) /(1-R)] \gamma\} X
$$

where the first part of the constant just contains the cavity parameters and the second part is the OGE coefficient. Equation (15) shows the coefficient $X$ as dependent on the relative temperatures of the discharge tube walls and the extra temperature generated by the gas discharge, and should thus be dependent on the level of the discharge 
excitation (described by the discharge $P / L$ ) and the gas composition and total pressure. In this section we examine in detail the behaviour of the coefficient $X$ with variation of discharge $P / L$, wall temperature and gas pressure, and in $\S 3.4$ below we look at variations in cavity parameters affecting the first part of the constant in equation (20).

The gas temperature in an electrical discharge can be calculated but only with some difficulty in a gas mixture with a radial current gradient (see for instance Laderman and Byron 1971). We have previously shown how a simple thermocouple technique can be used to accurately measure gas temperatures in $\mathrm{CO}$ laser mixtures and the technique is very effective at determining small temperature differences caused by gas mixture changes (Smith 1977). Furthermore it has been shown (Laderman and Byron 1971, Shields and Smith 1977) that the discharge generated temperature difference between the tube axis and the confining wall surface is dependent only on the discharge $P / L$. It does not vary with the gas total pressure (except at pressures considerably lower than those of concern here). However the temperature difference is very dependent on the gas mixture, because of thermal conductivity variations. In particular a typical laser mixture (with composition of 20 to $50 \%$ medium molecular weight components, $\mathrm{CO}_{2}$, $\mathrm{N}_{2}, \mathrm{CO}$ ) will be hotter than helium on its own, and the addition of a heavy molecular weight component such as xenon increases the temperature further. We have extended our measurements to sealed $\mathrm{CO}_{2}$ laser mixtures of varying composition (with and without added xenon) and all the gas temperatures used in our calculations are based on these actual measurements, with the $t_{\mathrm{dis}}$ used being the radially averaged value $\left(0.6\left(t_{\mathrm{axis}}-\right.\right.$ $\left.t_{\text {wall }}\right)$-see Shields and Smith 1977).

For a constant gas mixture ( 3 Torr Xe, 3 Torr $\mathrm{CO}_{2}, 4$ Torr $\mathrm{N}_{2}, 10$ Torr $\mathrm{He}$ ) the voltage fluctuation $(\Delta V)$ across the tube was recorded as the cavity was alternately blocked and unblocked to produce the maximum variation of $P_{\text {out }}$ (varying with current, $6.6 \mathrm{~W}$ at $16 \mathrm{~mA}$, but only $1.3 \mathrm{~W}$ at $2 \mathrm{~mA}$ and $1.4 \mathrm{~W}$ at $38 \mathrm{~mA}$ ). The experimental values for the OGE coefficient $X$ in figure 3 were then calculated from

$$
X=\left(\mathrm{i} \Delta V / \gamma P_{\text {out }}\right)[(1-R) /(1+R)]
$$

with $\gamma$ taken to be 0.15 and $R=0.9$. Also shown is a theoretical curve for $X$, obtained from equation (15) with a cavity wall temperature of $280 \mathrm{~K}$ and $t_{\mathrm{dis}}$ measured as described above (increasing from $75 \mathrm{~K}$ at $P / L=1 \mathrm{~W} \mathrm{~cm}^{-1}$ to $180 \mathrm{~K}$ at $3 \mathrm{~W} \mathrm{~cm}^{-1}$ ). There is a good agreement in the absolute value of the coefficient $X$ obtained by the two methods and reasonable agreement in the form of the variation of $X$ with $P / L$. The decrease of the

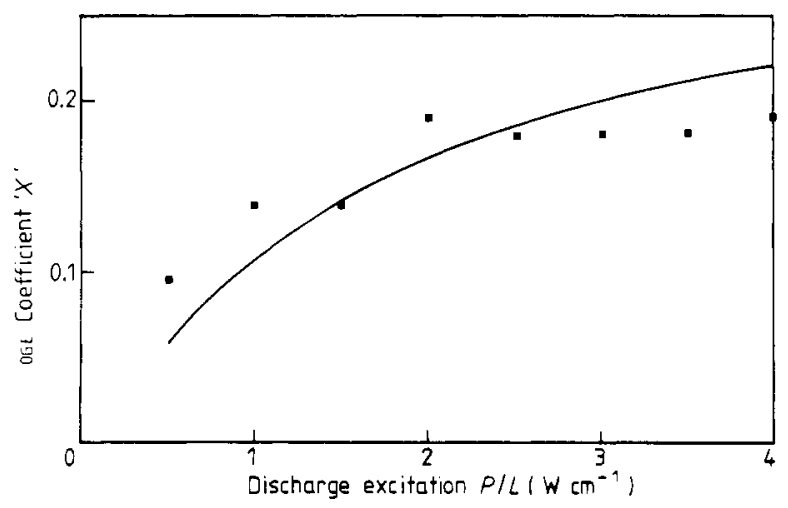

Figure 3. Variation of OGE coefficient $X$ with discharge excitation $P / L$ in 3 Torr Xe, 3 Torr $\mathrm{CO}_{2}, 4$ Torr $\mathrm{N}_{2}, 10$ Torr He. 


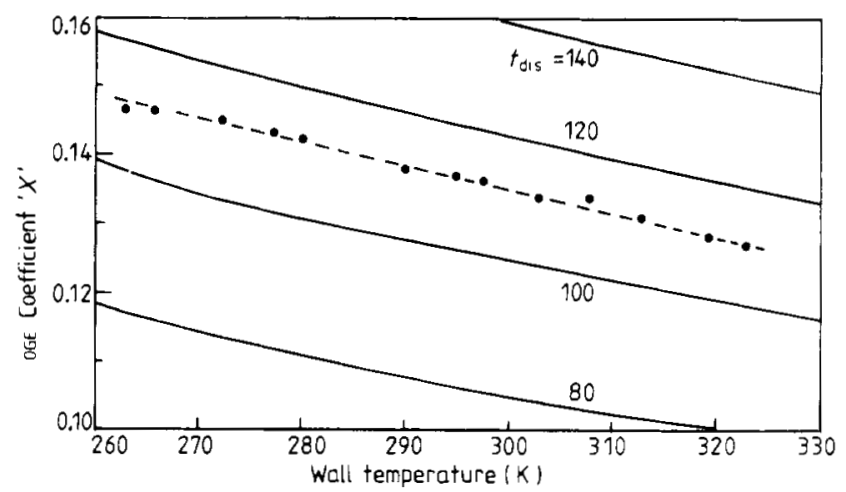

Figure 4. Variation of OGE coefficient $X$ with wall temperature for a constant current of 10 $\mathrm{mA}$ in 1 Torr Xe, 2.5 Torr $\mathrm{CO}_{2}, 3.5$ Torr $\mathrm{N}_{2}, 15$ Torr He. Solid curves show theoretical predictions for $t_{\text {dis }}$ from 80 to $140 \mathrm{~K}$.

slope of the $X$ against $P / L$ temperature derived curve at higher values of current is due to a decrease in the slope of the $t_{\text {dis }}$ against $P / L$ curve with increased excitation, and the slight misfit of the form of the curve could represent a change in the current radial profile with level of excitation relative to the radiation field profile (spot size).

As an alternative to varying the discharge generated temperature difference $t_{\mathrm{dis}}$ to produce variation of $X$, the wall temperature $t_{\text {wall }}$ has been changed by altering the temperature of the water in the cooling jacket surrounding the discharge tube. Figure 4 shows experimentally determined values of the OGE coefficient (measured as $\Delta P / \gamma I$ ) for values of the wall temperature from 263 to $323 \mathrm{~K}$ for a constant current of $10 \mathrm{~mA}$ in a mixture of 1 Torr Xe, 2.5 Torr $\mathrm{CO}_{2}, 3.5$ Torr $\mathrm{N}_{2}$ and 15 Torr He. Also shown are theoretical values of $X$ based on equation (15) for assumed discharge generated temperature differences $t_{\text {dis }}$ of $80,100,120$ and $140 \mathrm{~K}$. Clearly the form of the variation of $\Delta P / \gamma I$ with wall temperature is as predicted and a gas temperature of $\sim 110 \mathrm{~K}$ is indicated. whereas the predicted $t_{\text {dis }}$ for this discharge excitation and gas is $105 \pm 10 \mathrm{~K}$. indicating excellent absolute agreement for the theory.

Another way of varying the gas temperature, as an alternative to varying the discharge current or the wall temperature, is to vary the gas pressure. We have excited a $10 \mathrm{~mA}$ discharge in a constant gas mixture of $6 \% \mathrm{Xe}, 6 \% \mathrm{CO}_{2}, 12 \% \mathrm{~N}_{2}, 76 \% \mathrm{He}$ and figure 5 shows the experimentally determined values of the OGE coefficient $X$ over the pressure range of 10 to 24 Torr. As previously, the $X$ values are calculated from the $\Delta P / P_{\text {out }}$ results using equation (18). There is a significant increase in the magnitude of $X$ with increase in total pressure.

An increase is to be expected because (with constant current excitation) the voltage per unit length will increase with pressure and hence the excitation per unit length $P / L$ will increase (see equation (15)). The broken curve shows the theoretical values of $X$ predicted by equation (15), with an increase of $t_{\text {dis }}$ due to the increasing $P / L$ with increasing pressure. Although the amplitude of the theoretical prediction agrees reasonably with the empirical values, the rate of increase of the theoretical $X$ with pressure is not so rapid. Implicit in the derivation of the theoretical curve is the assumption that the radial current profile and the temperature profile do not change. Now with variation of the gas pressure from 10 to 24 Torr in an $8 \mathrm{~mm}$ bore tube, one is in a transitional region from a wall-dominated recombination discharge (at low pressures) to a bulk-gas recombination controlied discharge and the discharge will be increasingly constricted as the pressure is increased. Thus the current profile will no longer follow the form of the 


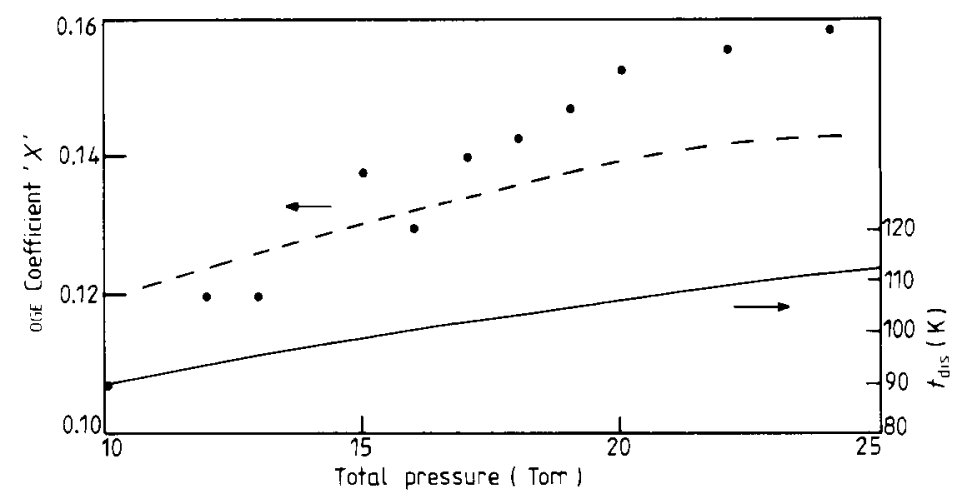

Figure 5. Variation of OGE coefficient $X$ with total gas pressure for a $10 \mathrm{~mA}$ discharge in $6 \%$ $\mathrm{Xe}, 6 \% \mathrm{CO}_{2}, 12 \% \mathrm{~N}_{2}, 76 \% \mathrm{He}$. Broken curve shows theoretical prediction, assuming the experimentally determined values of $t_{\text {dis }}$ (full curve).

zeroth-order Bessel function and the assumed radially averaged value of the gas temperature $\left(t_{\text {axis }}-t_{\text {wall }}\right)$ will no longer be constant with a value of $0.6\left(t_{\text {axis }}-t_{\text {wall }}\right)$ (Schwartz and Margalith 1974). The exact magnitude of this second order effect is difficult to accurately determine, but as the discharge constricts so the gas will be relatively hotter on the discharge tube axis, where the modal field (assuming a TEM $_{00 n}$ mode) is maximum and hence the calculated value will become increasingly too small as the pressure increases and the discharge constricts. Such a pressure variation is apparent in the experimental results of figure 5 .

\subsection{Intra-and extra-cavity perturbation effects}

To further examine the dependence of the discharge power fluctuation on the laser power consider equation (20) again. It shows that the normalised discharge power fluctuation $\Delta P / P_{\text {out }}$ should depend not only on the magnitude of the OGE coefficient $X$. but also on the cavity dependent factor $\gamma(1+R) /(1-R)$. As the output coupler reflectivity is increased $(1+R) /(1-R)$ will increase, but the total cavity losses $\gamma$ will decrease.

First consider the effect of varying the output mirror reflectivity. Figure 6 shows the variation of the experimentally determined discharge power change $\Delta P$ with discharge

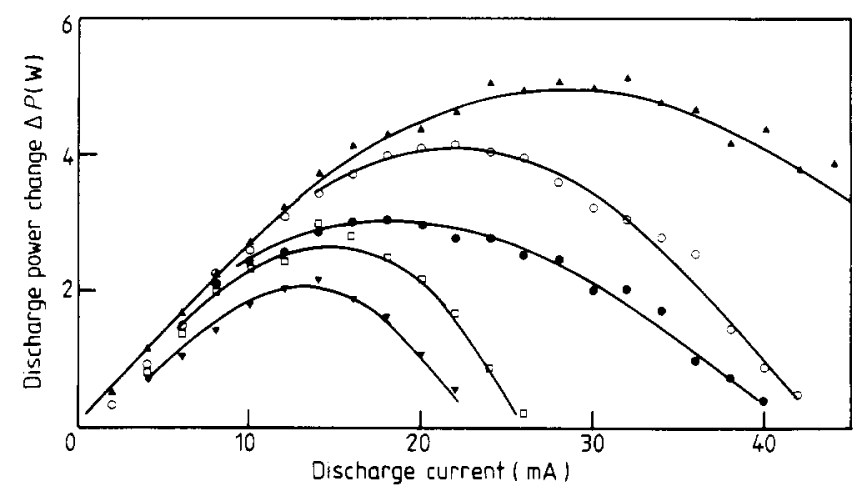

Figure 6. Variation of discharge power change with discharge current for output coupling reflectivities from 80 to $98 \%$ with a 3 Torr $\mathrm{Xe}, 3$ Torr $\mathrm{CO}_{2}, 4$ Torr $\mathrm{N}_{2}, 16$ Torr He mixture: $\boldsymbol{\nabla}, 80 \%, \square, 85 \% ; 0,90 \% ; 0.94 \% ; \boldsymbol{\Lambda}, 98 \%$. 
current for a fixed gas mixture ( 3 Torr Xe, 3 Torr $\mathrm{CO}_{2}, 4$ Torr $\mathrm{N}_{2}, 16$ Torr $\mathrm{He}$ ) for a range of different coupling mirrors with reflectivities from $80 \%$ to $98 \%$. The increase of $\Delta P$ with $R$ reflects the increase of the intra-cavity irradiance as more of the stimulated emission is retained within the cavity rather than contributing to an output laser beam.

The results of figure 6 (along with the laser power output results) are shown in figure 7 as the variation of $\Delta P / P_{\text {out }}$ with mirror reflectivity $R$. (In each case with $\Delta P$ being determined as the laser power $P_{\text {out }}$ is switched from its maximum value to zero, by blocking the cavity radiation field.) Clearly with the lower values of $R$ the portion of the cavity losses $\gamma$ due to the output coupling $(1-R)$ is considerable and the variations of $(1+R) /(1-R)$ and $\gamma$ tend to cancel one another out and $\Delta P / P_{\text {out }}$ only varies slowly with $R$. But at high values of $R$ the fixed (non-output coupling) losses contribution to $\gamma$ becomes important and $\Delta P / P_{\text {out }}$ increases rapidly with $R$.

A measure of the magnitude of the intra-cavity losses can be determined as follows. With a knowledge of the value of the OGE coefficient $X$ calculated for the discharge conditions and gas mixture from equation (15) and the experimentally determined values of $\Delta P / P_{\text {out }}$, the values of $\gamma$ for different values of $R$ are obtained from equation $(20)$, and these are plotted in figure 7 . The intra-cavity losses are just $\gamma-(1-R)$, and these are also plotted in figure 7. We have used the manufacturers' values for the various mirror reflectivities $R$ (and the probable uncertainties in these produce the rather large error bars as shown). There is a considerable spread in the derived values of $\gamma-$ $(1-R)$, from 0.02 to 0.07 ; with a most probable value of about 0.05 , and this is the value which we have assumed in $\$ \S 3.2$ and 3.3 above.

Now although it is convenient to express the radiation field in terms of the easily measured laser output power $P_{\text {out }}$, it is desirable to examine in more detail the power lost from the molecular system and transferred by stimulated emission to the cavity radiation field. In the case of an actual laser oscillator this $P_{\text {lost }}$ (osc) is dissipated as both the laser beam and in internal cavity losses, and is given by equation (12) in terms of the cavity irradiance, which is related to $P_{\text {out }}$ by equation (17),

$$
P_{\text {lost }}(\mathrm{Osc})=\frac{1}{2} I \gamma A=\frac{1}{2} \gamma[(1+R) /(1-R)] P_{\text {out }}
$$

and hence $P_{\text {lost }}$ (OSC) can be determined from $P_{\text {out }}$ using the established values of the cavity parameters $R$ and $\gamma$.

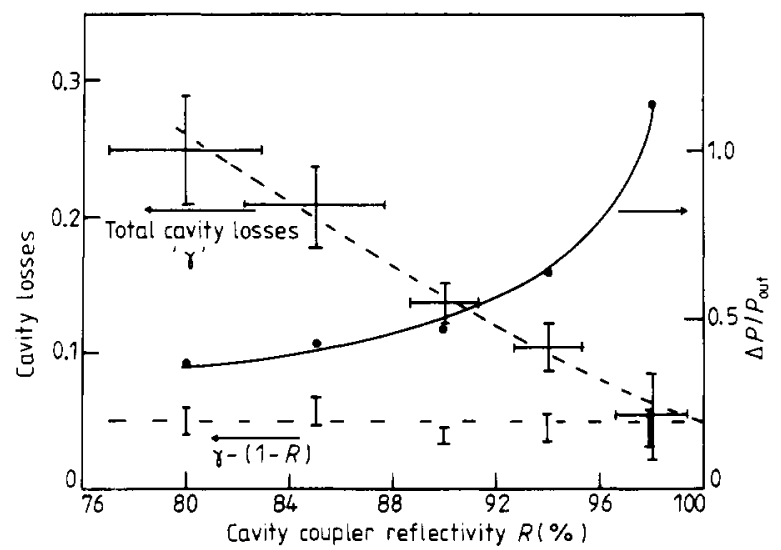

Figure 7. Variation with cavity coupler reflectivity of total cavity losses $\gamma$, intra-cavity losses $\gamma-(1-R)$ and normalised discharge power change $\Delta P / P_{\text {our }}$. Gas mixture 3 Torr Xe, 3 Torr $\mathrm{CO}_{2}, 4$ Torr $\mathrm{N}_{2}, 16$ Torr He. 
$P_{\text {lost }}$ (osc) is related to $\Delta P$ by equation (22) and equations (15) and (20) to give

$$
P_{\text {lost }}(\text { osc })=\Delta P / 2 X=\Delta P\left(t_{\mathrm{w} / \mathrm{dis}} / 1\right)
$$

and so there should be a linear relationship between $P_{\text {lost }}(\mathrm{Osc})$ and the discharge power change $\Delta P$.

Furthermore if one considers not a laser oscillator, but a separate laser amplifier tube, then the power lost $P_{\text {lost }}$ (amp) to a radiation beam (area $A$, irradiance $I$ ) passing through the amplifier tube will just be $\alpha L A I \mathrm{e}^{\alpha \mathrm{L}}$ where $\alpha$ is the medium gain coefficient. If the product $\alpha L$ is small then

$$
P_{\text {lost }}(\mathrm{amp})=\alpha L A I
$$

and for the amplifier tube, from (10)-(15) and (24)

$$
P_{\text {lost }}(\mathrm{amp})=\Delta P / 2 X=\Delta P\left(t_{\mathrm{w} /} / t_{\mathrm{dis}}+1\right) \text {. }
$$

Thus it should be possible to examine the predicted relationship between $\Delta P$ and $P_{\text {lost }}$ over a large range of irradiance levels, with large values inside a laser cavity with $P_{\text {lost }}$ (osc) being determined from $P_{\text {out }}$ by equation (23) and small values with a !aser beam passing through an amplifier cell with $P_{\text {lost }}(\mathrm{amp})$ being determined from equation (25).

Experimentally an amplifier gain cell $(9.3 \mathrm{~cm}$ gain length) was constructed and operated in exactly the same manner as the tube inside the laser oscillator, with a $10 \mathrm{~mA}$ discharge in a gas mixture of 1 Torr Xe, 3 Torr $\mathrm{CO}_{2}, 4$ Torr $\mathrm{N}_{2}, 16$ Torr He. Figure 8 shows the experimentally measured power perturbation $\Delta P$ in the amplifier and the corresponding values of $P_{\text {lost }}(\mathrm{amp}$ ) determined from equation (24) (for known values of beam probe power and a measured small signal gain of $0.005 \mathrm{~cm}^{-1}$ ). Also shown are the discharge power perturbations $\Delta P$ in the oscillator and the corresponding values of $P_{\text {lost }}($ osc) determined from equation (22).

The solid line in figure 8 is the theoretical curve determined from both equations (23) and (25) assuming a $t_{\text {dis }}$ of $110 \mathrm{~K}$. This temperature is in good agreement with the expected $105 \pm 10 \mathrm{~K}$. The close correspondence of both the oscillator and amplifier values over four orders of magnitude with the theoretical expectation is very satisfactory.

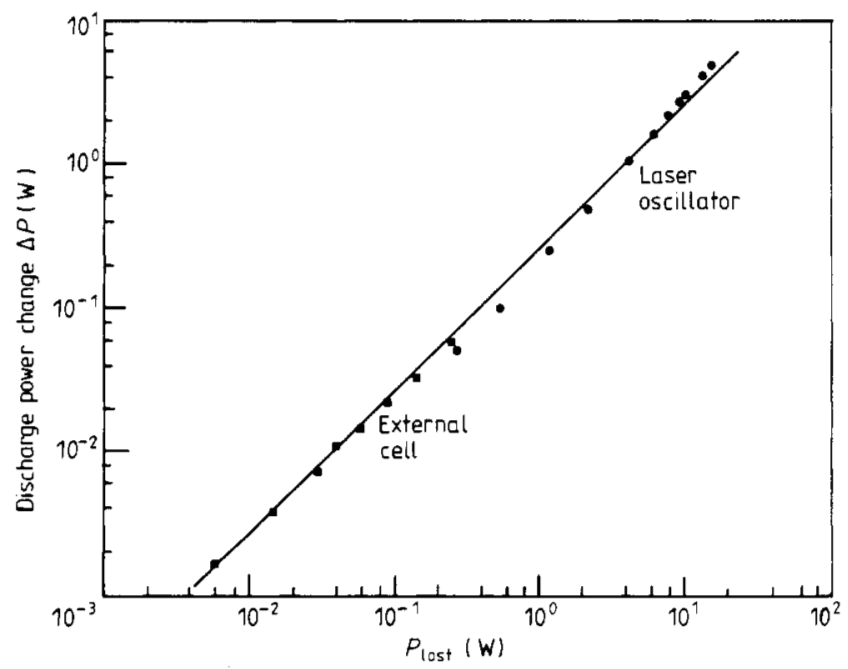

Figure 8. Variation of discharge power change with $P_{\text {lost }}$ for a $10 \mathrm{~mA}$ discharge in 1 Torr Xe, 3 Torr $\mathrm{CO}_{2}, 4$ Torr $\mathrm{N}_{2}, 16$ Torr He both internally in a laser oscillator and externally in a gain cell. 


\section{Conclusions}

A simple power perturbation model has been developed based on the change of the gas number density in the discharge caused by the variation in gas heating as the stimulated radiation field provides an alternative decay path to $\mathrm{V}-\mathrm{V}-\mathrm{T}$ upper laser level relaxation. The number density changes lead directly to collisional changes between the electrons and molecules: but it is only necessary to assume change in the number of collisions, not change in the energy exchange per collision.

The model has been kept relatively simple by assuming radially constant values of the radiation field and electron density and assuming an average gas temperature value across the tube profile of $0.6\left(t_{\mathrm{dis}}-t_{\mathrm{wall}}\right)$. There is excellent agreement between the observed discharge power perturbation $\Delta P$ and the predicted values for several different but typical sealed laser mixtures operating under typical laser conditions. However some discrepancy is evident when effects due to changes in the discharge radial profile might be expected to be strong. Thus in $\$ 3.3$ (figure 3 ) it was observed that the value of the OGE coefficient $X$ varied less with the degree of discharge excitation $P / L$ than the simple theory predicted, and also in $\$ 3.3$ (figure 5) it was evident that the coefficient $X$ varied more than the theoretical prediction as the gas pressure was increased. Both these effects are at least qualitatively commensurate with a small change of the radial current profile.

Although this theory has been developed specifically to account for discharge perturbation effects, induced by radiation field changes in the fundamental $00^{\circ} 1 \rightarrow\left[10^{\circ} 0\right.$, $\left.02^{\circ} 0\right]_{1},{ }_{11}$ laser transitions, it should also be directly applicable to all discharge systems where gas heating effects are considerable and the exact form of the discharge $\mathrm{V}-\mathrm{i}$ characteristic is wholly or largely controlled by temperature effects. Thus the theory should be applicable to sequence band $\mathrm{CO}_{2}$ laser discharges, $\mathrm{CO}$ laser discharges and the numerous molecular systems where the OGE is now being applied as a spectroscopic tool.

\section{Acknowledgments}

This work was supported by the SERC and we would like to thank Mr Fritz Akerboom and Mr Norrie Campbell for invaluable technical assistance.

\section{References}

Ferguson A I 1982 Phil. Trans. R. Soc. A $307645-57$

Foote P D and Mohler F L 1925 Phys. Rev. 26 195-207

Goldsmith J E M and Lawler J E 1981 Contemp. Phys. 22 235-48

Laderman A J and Byron S R 1971 J. Appl. Phys. 42 3138-44

Meissner K W and Graffunder W 1927 Ann. Phys., Lpz $841009-46$

Moffatt S and Smith A L S 1981 Opt. Comm. 37 119-22

Penning F M 1928 Physica 8 137-40

Shields H and Smith A L S 1977 J. Appl. Phys. 48 4807-8

Schwartz J and Margalith E 1974 J. Appl. Phys. 45 4469-76

Smith A L S 1977 IEEEJ. Quantum Electron. QE-13 59-61

Smith A L S and Brooks M 1979 J. Phys. D: Appl. Phys. 12 1237-48

Smith A L S and Mellis J 1984 to be published

Smith A L S and Moffatt S 1979 Opt. Comm. 30 213-8 